\title{
Vickrey Auctions for Irregular Distributions
}

\author{
Balasubramanian Sivan ${ }^{1}$ and Vasilis Syrgkanis ${ }^{2}$ \\ 1 Microsoft Research, bsivanemicrosoft.com \\ 2 Computer Science Dept., Cornell University, vasilis@cs. cornell.edu
}

\begin{abstract}
The classic result of Bulow and Klemperer [1] says that in a singleitem auction recruiting one more bidder and running the Vickrey auction achieves a higher revenue than the optimal auction's revenue on the original set of bidders, when values are drawn i.i.d. from a regular distribution. We give a version of Bulow and Klemperer's result in settings where bidders' values are drawn from non-i.i.d. irregular distributions. We do this by modeling irregular distributions as some convex combination of regular distributions. The regular distributions that constitute the irregular distribution correspond to different population groups in the bidder population. Drawing a bidder from this collection of population groups is equivalent to drawing from some convex combination of these regular distributions. We show that recruiting one extra bidder from each underlying population group and running the Vickrey auction gives at least half of the optimal auction's revenue on the original set of bidders.
\end{abstract}

Keywords: Bulow-Klemperer, irregular distributions, prior-independent, Vickrey auction

\section{Introduction}

Simplicity and detail-freeness are two much sought-after themes in auction design. The celebrated classic result of Bulow and Klemperer [1] says that in a standard singleitem auction with $n$ bidders, when the valuations of bidders are drawn i.i.d from a distribution that satisfies a regularity condition, running a Vickrey auction (second-price auction) with one extra bidder drawn from the same distribution yields at least as much revenue as the optimal auction for the original $n$ bidders. The Vickrey auction is both simple and detail-free since it doesn't require any knowledge of bidder distributions. Given this success story for i.i.d. regular distributions, we ask in this paper, what is the analogous result when we go beyond i.i.d regular settings? Our main result is a version of Bulow and Klemperer's result to non-i.i.d irregular settings. Our work gives the first positive results in designing simple mechanisms for irregular distributions, by parameterizing irregular distributions, i.e., quantifying the amount of irregularity in a distribution. Our parameterization is motivated by real world market structures and in turn indicates that most realistic markets will not be highly irregular with respect to this metric. Our results enable the first positive approximation bounds on the revenue of the second-price auction with an anonymous reserve in both i.i.d. and non-i.i.d. irregular settings.

Before explaining our results, we briefly describe our setting. We consider a singleitem auction setting with bidders having quasi-linear utilities. That is the utility of a 
bidder is his value for the item if he wins, less the price he is charged by the auction. We study auctions in the Bayesian setting, i.e. the valuations of bidders are drawn from known distribution 3 . We make the standard assumption that bidder valuations are drawn from independent distributions.

Irregular distributions are common. The technical regularity condition in Bulow and Klemperer's result is quite restrictive, and indeed irregular distributions are quite common in markets. For instance, any distribution with more than a single mode violates the regularity condition. The most prevalent reason for a bidder's valuation distribution failing to satisfy the regularity condition is that a bidder in an auction is randomly drawn from a heterogeneous population. The population typically is composed of several groups, and each group has its characteristic preferences. For instance the population might consist of students and seniors, with each group typically having very different preferences from the other. While the distribution of preferences within any one group might be relatively well-aligned and the value distribution might have a single mode and satisfy the regularity condition, the distribution of a bidder drawn from the general population, which is a mixture of such groups, is some convex combination of these individual distributions. Such a convex combination violates regularity even in the simplest cases.

For a variety of reasons, including legal reasons and absence of good data, a seller might be unable to discriminate between the buyers from different population groups and thus has to deal with the market as if each buyer was arriving from an irregular distribution. However, to the least, most sellers do know that their market consists of distinct segments with their characteristic preferences.

Measure of Irregularity. The above description suggests that a concrete measure of irregularity of a distribution is the number of regular distributions required to describe it. We believe that such a measure could be of interest in both designing mechanisms and developing good provable revenue guarantees for irregular distributions in many settings. It is a rigorous measure of irregularity for any distribution since any distribution can be well-approximated almost everywhere by a sufficient number of regular ones and if we allow the number of regular distributions to grow to infinity then any distribution can be exactly described Irregular distributions that typically arise in practice are combinations of a small number of regular distributions and this number can be considered almost a constant with respect to the market size. In fact there exist evidence in recent [8, 6] and classical [12] microeconomic literature that irregularity of the value distribution predominantly arises due to market segmentation in a small number of parts (e.g. loyal customers vs. bargain-hunters [8], luxury vs. low income buyers [6] etc). Only highly pathological distributions require a large number of regular distributions to be described — such a setting in a market implies that the population is heavily segmented and each segment has significantly different preferences from the rest.

\footnotetext{
${ }^{3}$ One of the goals of this work is to design detail-free mechanisms, i.e., minimize the dependence on knowledge of distributions. Thus most of our results make little or no use of knowledge of distributions. We state our dependence precisely while stating our results.

${ }^{4}$ This follows from the fact that a uniform distribution over an interval is a regular distribution and every distribution can be approximated in the limit using just uniform distributions.
} 
Motivated by this, we consider the following setting: the market/population consists of $k$ underlying population groups, and the valuation distribution of each group satisfies the regularity condition. Each bidder is drawn according to some probability distribution over these groups. That is bidder $i$ arrives from group $t$ with probability $p_{i, t}$. Thus if $F_{t}$ is the cumulative distribution function (cdf) of group $t$, the cdf of bidder $i$ is $F_{i}=\sum_{t} p_{i, t} F_{t}$. For example, consider a market for a product that consists of two different population groups, say students and seniors. Now suppose that two bidders come from two cities with different student to senior ratios. This would lead to the probability $p_{i, t}$ 's to be different for different $i$ 's. This places us in a non-i.i.d. irregular setting. All our results also extend to the case where these probabilities $p_{i, t}$ are arbitrarily correlated.

Example 1 (An illustrative example). Consider an eBay seller of an ipad. One could think of the market as segmented mainly in two groups of buyers: young and elder audience. These two market segments have completely different value distributions. Suppose for instance, that the value distribution of young people is distributed as a normal distribution $N\left(\mu_{y}, \sigma\right)$ while the elder's is distributed as a normal distribution $N\left(\mu_{e}, \sigma\right)$ with $\mu_{y}>\mu_{e}$. In addition, suppose that the eBay buyer population is composed of a fraction $p_{y}$ young people and $p_{e}<p_{y}$ of elders. Thus the eBay seller is facing an irregular valuation distribution that is a mixture of two Gaussian distribution with mixture probabilities $p_{y}$ and $p_{e}$ (see Figure 1). Even more generally, this mixture could be dependent on the city of the buyer and hence be different for different buyers.

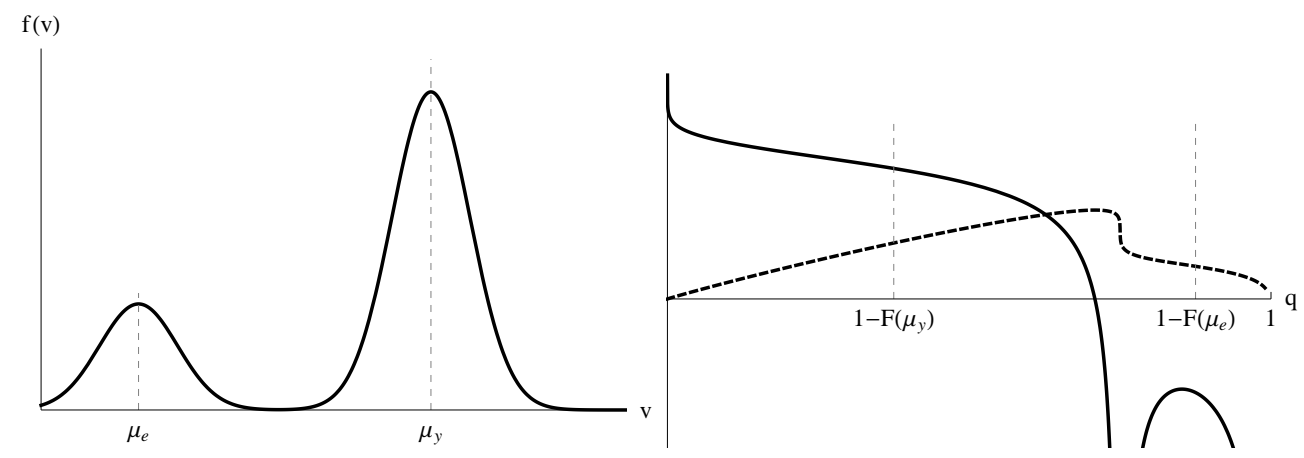

Fig. 1. Left figure depicts pdf of the bimodal distribution of valuations of Example 1 while the right figure depicts the revenue (dashed) $R(q)=q \cdot F^{-1}(1-q)$ where $q$ is the probability of sale, and the marginal revenue curve $\frac{d R(q)}{d q}$ for this distribution.

The eBay seller has two ways of increasing the revenue that he receives: a) increasing the market competition by bringing extra bidders through advertising (possibly even targeted advertising), and b) setting appropriately his reserve price in the second price auction that he runs. Observe that he has no means of price discriminating. Even running Myerson's auction which is non-discriminatory for i.i.d. settings leads to randomization. In particular, randomization leads to the undesirable feature of sometimes serving 
an agent with smaller value. This raises the main questions that we address in this paper: how should he run his advertising campaign? How many people more (either through targeted or non-targeted advertising) should he bring to the auction to get a good approximation to the optimal revenue? What approximation of the optimal revenue is he guaranteed by running a Vickrey auction with a single anonymous reserve?

Giving a sneak preview of our main results, our paper gives positive results to all the above questions: 1) bringing just one extra young bidder in the auction (targeted advertising) and running a Vickrey auction with no reserve would yield revenue at least $1 / 2$ of the optimal revenue (Theorem 2 2,2 ) bringing 2 extra bidders drawn from the distribution of the combined population (non-targeted advertising) would yield at least $\frac{1}{2}\left(1-\frac{1}{e}\right)$ of the optimal revenue (Theorem [4), 3) running a Vickrey auction among the original $n$ bidders with an anonymous reserve price can yield an 8-approximation of the optimal revenue (Theorem 5).

\section{Our Results}

First result (Section 3): Targeted Advertising for non-i.i.d. irregular settings. We show that by recruiting an extra bidder from each underlying group and running the Vickrey auction, we get a revenue that is at least half of the optimal auction's revenue in the original setting. While the optimal auction is manifestly impractical in a non-i.i.d. irregular setting due to its complicated rules, delicate dependence on knowledge of distribution and its discriminatory nature 5 , the Vickrey auction with extra bidders is simple and detail-free: it makes no use of the distributions of bidders. The auctioneer must just be able to identify that his market is composed of different groups and must conduct a targeted advertising campaign to recruit one extra bidder from each group. This result can be interpreted as follows: while advertising was the solution proposed by Bulow and Klemperer [1] for i.i.d. regular distributions, targeted advertising is the right approach for non-i.i.d. irregular distributions.

Tightness. While we do not know if the the factor 2 approximation we get is tight, Hartline and Roughgarden [7] show that even in a non-i.i.d. regular setting with just two bidders it is impossible to get better than a 4/3-approximation by duplicating the bidders, i.e., recruiting $n$ more bidders distributed identically to the original $n$ bidders. This lower bound clearly carries over to our setting also: there are instances where recruiting only one bidder from each different population group cannot give anything better than a 4/3-approximation.

Second result (Main result, Section 4): Just one extra bidder for hazard rate dominant distributions. If the $k$ underlying distributions are such that one of them stochastically dominates, hazard-rate wise, the rest, then we show that recruiting just one extra bidder from the hazard rate dominant distribution and running the Vickrey auction gets at

\footnotetext{
${ }^{5}$ The optimal auction in a non-i.i.d. setting will award the item to the bidder with the highest virtual value and this is not necessarily the bidder with the highest value. In addition, typically a different reserve price will be set to different bidders. This kind of discrimination is often illegal or impractical. Also, the exact form of the irregular distribution will determine which region's of bidder valuations will need to be "ironed", i.e. treated equally.
} 
least half of the optimal revenue for the original setting. A distribution $F$ hazard rate dominates a distribution $G$ iff for every $x$ in the intersection of the support of the two distributions the hazard rate $h_{F}(x)\left(=\frac{f(x)}{1-F(x)}\right.$, where $f(\cdot)$ and $F(\cdot)$ are the pdf and cdf respectively) is at most $h_{G}(x)\left(=\frac{g(x)}{1-G(x)}\right.$, where $g(\cdot)$ and $G(\cdot)$ are the pdf and cdf respectively). We denote such a domination by $F \succeq_{h r} G$.

Further, hazard rate dominance requirement is not uncommon: for instance, if all the $k$ underlying distributions were from the same family of distributions like the uniform, exponential, Gaussian or even power law, then one of them is guaranteed to hazard rate dominate the rest. Though several common distributions satisfy this hazard rate dominance property, it has never been previously exploited in the context of approximately optimal auctions.

Third result (Section [5): Non-targeted advertising for i.i.d. irregular distributions. When the bidders are identically distributed, i.e., the probability $p_{i, t}$ of distribution $t$ getting picked for bidder $i$ is the same for all $i$ (say $p_{t}$ ), we show that if each $p_{t} \geq \delta$, then bringing $\Theta\left(\frac{\log (k)}{\delta}\right)$ extra bidders drawn from the original distribution (and not from one of the $k$ underlying distributions) yields a constant approximation to the optimal revenue. Further in the special case where one of the underlying regular distributions hazard rate dominates the rest and its mixture probability is $\delta$ then $\Theta\left(\frac{1}{\delta}\right)$ bidders drawn from the original distribution are enough to yield a constant approximation. This shows that when each of the underlying population groups is sufficiently thick, then recruiting a few extra bidders from the original distribution is all that is necessary.

Remark 1. For the latter result it is not necessary that the decomposition of the irregular distribution that we use should resemble the actual underlying population groups. Even if the market is highly fragmented with several population groups, as long as there is mathematically some way to decompose the irregular distribution into the convex combination of a few regular distributions our third result still holds.

Fourth result (Section 6): Vickrey with a Single (Anonymous) Reserve. Suppose we are unable to recruit extra bidders. What is the next simplest non-discriminatory auction we could hope for? The Vickrey auction with a single reserve price. We show that when the non-i.i.d irregular distributions all arise as different convex combinations of the $k$ underlying regular distributions, there exists a reserve such that the Vickrey auction with this single reserve obtains a $4 k$ approximation to the optimal revenue. Though the factor of approximation is not small, it is the first non-trivial approximation known for non-i.i.d irregular distributions via Vickrey with anonymous reserve. In addition, as we already explained, in typical market applications we expect the number of different population groups $k$ to be some small constant.

What is the best one can hope for a non-i.i.d irregular setting? Chawla, Hartline and Kleinberg [2] show that for general non-i.i.d irregular distributions it is impossible to get a $o(\log n)$ approximation using Vickrey auction with a single reserve price, and it is unknown if this lower bound is tight, i.e., we do not yet know of a $\Theta(\log n)$ approximation. However the $o(\log n)$ impossibility exists only for arbitrary non-i.i.d irregular settings and doesn't apply when you assume some natural structure on the irregularity of the distributions, which is what we do. 
To put our results in context: Single reserve price Vickrey auctions were also analyzed by Hartline and Roughgarden [7] for non-i.i.d regular settings, that showed that there exists a single reserve price that obtains a 4-approximation. Chawla et al. [3] show that when bidders are drawn from non-i.i.d irregular distributions, a Vickrey auction with a distribution-specific reserve price obtains a 2-approximation. Thus if there are $k$ different distributions, $k$ different reserve prices are used in this result. This means that if we insist on placing a single (anonymous) reserve price, this result guarantees a $O(1 / k)$ approximation. In particular, when all distributions are different, i.e. $k=n$, this boils down to a $O(1 / n)$ approximation.

In contrast, our result shows that even when all the distributions are different, as long as every irregular distribution can be described as some convex combination of $k$ regular distributions, Vickrey with a single reserve price gives a factor $4 k$ approximation. Further the factor does not grow with the number of players $n$.

Remark 2. We also show that if the bidders are distributed with identical mixtures and the mixture probability is at least $\delta$ then Vickrey auction with a single reserve achieves a $\Theta\left(1+\frac{\log (k)}{n \delta}\right)$ approximation. If one of the regular distribution hazard rate dominates the rest and has mixture probability $\delta$, then Vickrey with a single reserve achieves a $\Theta\left(1+\frac{1}{n \delta}\right)$ approximation.

Observe that if all $k$ regular distributions in the mixture have equal probability of arriving, then our results shows that a Vickrey auction with a single reserve achieves at least a $\Theta\left(1+\frac{k \log (k)}{n}\right)$ of the optimal revenue. This approximation ratio becomes better as the number of bidders increases, as long as the number of underlying regular distributions remains fixed. If the number of underlying distributions increases linearly with the number of bidders, then the result implies a $\Theta(\log (n))$ approximation, matching the lower bound of [3].

Related Work. Studying the trade-off between simple and optimal auctions has been a topic of interest for long in auction design. The most famous result is the already discussed result of Bulow and Klemperer [1] for single-item auctions in i.i.d regular settings. Hartline and Roughgarden [7] generalize [1]'s result for settings beyond singleitem auctions: they consider auctions where the set of buyers who can be simultaneously served form the independent set of a matroid; further they also relax the i.i.d constraint and deal with non-i.i.d settings. Dhangwatnotai, Roughgarden and Yan [5] study revenue approximations via VCG mechanisms with multiple reserve prices, where the reserve prices are obtained by using the valuations of bidders as a sample from the distributions. Their results apply for matroidal settings when the distributions are regular, and for general downward closed settings when the distributions satisfy the more restrictive monotone hazard rate condition. As previously discussed, Chawla et al. [3] show that for i.i.d irregular distributions, Vickrey auction with a single reserve price gives a 2-approximation to the optimal revenue and for non-i.i.d distributions Vickrey auction with a distribution-specific reserve price guarantees a 2-approximation; Chawla et al. [2] show that it is impossible to achieve a $o(\log n)$ approximation via Vickrey auction with a single reserve price for non-i.i.d irregular distributions. Single-item Vickrey auctions with bidder specific monopoly reserve prices were also studied in Neeman [11] 
and Ronen [13]. Approximate revenue maximization via VCG mechanisms with supply limitations were studied in Devanur et al. [4] and Roughgarden et al. [14].

\section{Preliminaries}

Basic model. We study single item auctions among $n$ bidders. Bidder $i$ has a value $v_{i}$ for a good, and the valuation profile for all the $n$ players together is denoted by $\mathbf{v}=$ $\left(v_{1}, v_{2}, \ldots, v_{n}\right)$. In a sealed bid auction each player submits a bid, and the bid profile is denoted by $\mathbf{b}=\left(b_{1}, b_{2}, \ldots, b_{n}\right)$. An auction is a pair of functions $(\mathbf{x}, \mathbf{p})$, where $x$ maps a bid vector to outcomes $\{0,1\}^{n}$, and $p$ maps a bid vector to $\mathbf{R}_{+}^{n}$, i.e., a nonnegative payment for each player. The players have quasi-linear utility functions, i.e., their utilities have a separable and linear dependence on money, given by $u_{i}\left(v_{i}, \mathbf{v}_{-i}\right)=$ $v_{i} x_{i}(\mathbf{v})-p_{i}(\mathbf{v})$. An auction is said to be dominant strategy truthful if submitting a bid equal to your value yields no smaller utility than any other bid in every situation, i.e., for all $\mathbf{v}_{-i}, v_{i} x_{i}(\mathbf{v})-p_{i}(\mathbf{v}) \geq v_{i} x_{i}\left(b_{i}, \mathbf{v}_{-i}\right)-p_{i}\left(b_{i}, \mathbf{v}_{-i}\right)$. Since we focus on truthful auctions in this paper $\mathbf{b}=\mathbf{v}$ from now on.

Distributions. We study auctions in a Bayesian setting, i.e., the valuations of bidders are drawn from a distribution. In particular, we assume that valuation of bidder $i$ is drawn from distribution $F_{i}$, which is independent from but not necessarily identical to $F_{j}$ for $j \neq i$. For ease of presentation, we assume that these distributions are continuous, i.e., they have density function $f_{i}$. We assume that the support of these distributions are intervals on the non-negative real line, with non-zero density everywhere in the interval.

Regularity and irregularity. The hazard rate function of a distribution is defined as $h(x)=\frac{f(x)}{1-F(x)}$. A distribution is said to have a Monotone Hazard Rate(MHR) if $h(x)$ is monotonically non-decreasing. A weaker requirement on distributions is called regularity: the function $\phi(x)=x-\frac{1}{h(x)}$ is monotonically non-decreasing. We do not assume either of these technical conditions for our distributions. Instead we assume that the market of bidders consists of $k$ groups and each group has a regular distribution $G_{i}$ over valuations. Each bidder is drawn according to some (potentially different) convex combination of these $k$ regular distributions, i.e., $F_{i}(x)=\sum_{t=1}^{k} p_{i, t} G_{t}(x)$. Such a distribution $F_{i}(\cdot)$ in most cases significantly violates the regularity condition.

In fact, mathematically, any irregular distribution can be approximated by a convex combination of sufficiently many regular distributions and as we take the number of regular distributions to infinity then it can be described exactly. Thus the number of regular distributions needed to describe an irregular distribution is a valid measure of irregularity that is well-defined for any distribution.

Revenue Objective. The objective in this paper to design auctions to maximize expected revenue, i.e., the expectation of the sum of the payments of all agents. Formally, the objective is to maximize $\mathbb{E}_{\mathbf{v}}\left[\sum_{i} p_{i}(\mathbf{v})\right]$. Myerson [10] characterized the expected revenue from any auction as its expected virtual surplus, i.e. the expected sum of virtual values of the agents who receive the item, where the virtual value of an agent is $\phi(v)=v-\frac{1}{h(v)}$. Formally, for all bidders $i, \mathbb{E}_{\mathbf{v}}\left[p_{i}(\mathbf{v})\right]=\mathbb{E}_{\mathbf{v}}\left[\phi_{i}\left(v_{i}\right) x_{i}(\mathbf{v})\right]$. The equality holds even if we condition on a fixed $v_{-i}$, i.e., $\mathbb{E}_{v_{i}}\left[p_{i}\left(v_{i}, \mathbf{v}_{-i}\right)\right]=\mathbb{E}_{v_{i}}\left[\phi\left(v_{i}\right) x_{i}\left(v_{i}, \mathbf{v}_{-i}\right)\right]$. 


\section{Targeted Advertising and the Non-i.i.d. Irregular Setting}

In this section we give our version of Bulow and Klemperer's result [1] for non-i.i.d irregular distributions.

Theorem 1. Consider an auction among $n$ non-i.i.d irregular bidders where each bidder's distribution $F_{i}$ is some mixture of $k$ regular distributions $\left\{G_{1}, \ldots, G_{k}\right\}$ (the set of regular distributions is the same for all bidders but the mixture probabilities could be different). The revenue of the optimal auction in this setting is at most twice the revenue of a Vickrey auction with $k$ extra bidders, where each bidder is drawn from a distinct distribution from $\left\{G_{1}, \ldots, G_{k}\right\}$.

Proof. Bidder $i$ 's distribution $F_{i}(x)=\sum_{t=1}^{k} p_{i, t} G_{t}(x)$ can be thought of as being drawn based on the following process: first a biased $k$-valued coin is flipped that decides from which distribution $G_{t}$ player $i$ 's value will come from (according to the probabilities $p_{i, t}$ ), and then a sample from $G_{t}$ is drawn. Likewise, the entire valuation profile can be thought of as being drawn in a similar way: first $n$ independent, and possibly non-identically biased, $k$-valued coin tosses, decide the regular distribution from each bidder's value is going to be drawn from. Subsequently a sample is drawn from each distribution.

Let the random variable $q_{i}$ be the index of the regular distribution that bidder $i$ 's value is going to be drawn, i.e., $q_{i}$ is the result of the coin toss for bidder $i$. Let $q$ denote the index profile of all players. Let $p(q)=\prod_{i=1}^{n} p_{i, q_{i}}$ be the probability that the index profile $q$ results after the $n$ coin tosses. Let $G(q)={ }^{\circ} G_{q_{i}}$ be the joint product distribution of players' values conditioned on the profile being $q$.

Let $M_{q}$ be the optimal mechanism when bidders' distribution profile is $q$. Let $\mathcal{R}_{M}^{q}$ be the expected revenue of mechanism $M_{q}$. Let $R_{M}^{q}(\mathbf{v})$ denote the revenue of the mechanism when bidders have value $\mathbf{v}$. The revenue of the optimal mechanism $M$ which cannot learn and exploit the actual distribution profile $q$ is upper bounded by the revenue of the optimal mechanism that can first learn $q$. Therefore we have,

$$
\mathcal{R}_{M} \leq \sum_{q \in[1 . . k]^{n}} p(q) \mathbb{E}_{\mathbf{v} \sim G(q)}\left[R_{M}^{q}(\mathbf{v})\right]
$$

Now, $\mathbb{E}_{\mathbf{v} \sim G(q)}\left[R_{M}^{q}(\mathbf{v})\right]$ corresponds to the optimal expected revenue when bidder $i$ 's distribution is the regular distribution $G_{q_{i}}$. Let $k(q)$ denote the number of distinct regular distributions contained in the profile $q$. Note that $k(q) \leq k$ for all $q$. Thus the above expectation corresponds to the revenue of a single-item auction where players can be categorized in $k(q)$ groups and bidders within each group $t$ are distributed i.i.d. according to a regular distribution $G_{t}$. Theorem 6.3 of [14] applies to such a setting and shows that the optimal revenue for each of these non-i.i.d regular settings will be at most twice the revenue of Vickrey auction with one extra bidder for each distinct distribution in the profile $q$. Hence,

$$
\begin{aligned}
\mathcal{R}_{M} \leq \sum_{q \in[1 . . k]^{n}} p(q) \mathbb{E}_{\mathbf{v} \sim G(q)}\left[R_{M}^{q}(\mathbf{v})\right] & \leq 2 \sum_{q \in[1 . . k]^{n}} p(q) \mathbb{E}_{\mathbf{v} \sim G(q)}\left[R_{S P_{n+k(q)}}(\mathbf{v})\right] \\
& \leq 2 \sum_{q \in[1 . . k]^{n}} p(q) \mathbb{E}_{\mathbf{v} \sim G(q)}\left[R_{S P_{n+k}}(\mathbf{v})\right]
\end{aligned}
$$


Since, the Vickrey auction with $k$ extra bidders doesn't depend on the index profile $q$ the RHS of (3) corresponds to the expected revenue of $S P_{n+k}$ when bidders come from the initial i.i.d irregular distributions.

The above proof actually proves an even stronger claim: the revenue from running the Vickrey auction with $k$ extra bidders is at least half approximate even if the auctioneer could distinguish bidders by learning the bidder distribution profile $q$ and run the corresponding optimal auction $R_{M}^{q}$.

Lower bound. A corner case of our theorem is when each bidder comes from a different regular distribution. From Hartline and Roughgarden [7] we know that a lower bound of $4 / 3$ exists for such a case. In other words there exists two regular distributions such that if the initial bidders came each from a different distribution among these, then adding two extra bidders from those distributions will not give the optimal revenue but rather a $4 / 3$ approximation to it. The same lower bound proves that if bidders came from the same mixture of these two regular distributions (i.e. are i.i.d), then the expected revenue of the auction that first distinguishes from which regular distribution each bidder comes from and then applies the optimal auction, yields higher revenue than adding two extra bidders from the two distributions and running a Vickrey auction.

\section{Just one extra bidder for hazard rate dominant distributions}

In this section we examine the setting where among the $k$ underlying regular distributions there exists one distribution that stochastically dominates the rest in the sense of hazard rate dominance. Hazard rate dominance is a standard dominance concept used while establishing revenue guarantees for auctions (see for example [9]) and states the following: A distribution $F$ hazard rate dominates a distribution $G$ iff for every $x$ in the intersection of the support of the two distributions: $h_{F}(x) \leq h_{G}(x)$. We denote such a domination by $F \succeq_{h r} G$.

In such a setting it is natural to ask whether adding just a single player from the dominant distribution is enough to produce good revenue guarantees. We actually show that adding only one extra person coming from the dominant distribution achieves exactly the same worst-case guarantee as adding $k$ extra bidders one from each underlying distribution.

Theorem 2. Consider an auction among $n$ non-i.i.d irregular bidders where each bidder's distribution $F_{i}$ is some mixture of $k$ regular distributions $\left\{G_{1}, \ldots, G_{k}\right\}$ such that $G_{1} \succeq_{h r} G_{t}$ for all $t$. The revenue of the optimal auction in this setting is at most twice the revenue of a Vickrey auction with one extra bidder drawn from $G_{1}$.

The proof is based on a new lemma for the regular distribution setting: bidders are drawn from a family of $k$ regular distributions such that one of them hazard-rate dominates the rest. This lemma can be extended to prove Theorem 2 in a manner identical to how Theorem 6.3 of Roughgarden et al. [14] was extended to prove Theorem 1 in our paper. We don't repeat that extension here, and instead just prove the lemma. The lemma uses the notion of commensurate auctions defined by Hartline and Roughgarden [7]. 
Lemma 1. Consider a non-i.i.d. regular setting where each player's value comes from some set of distributions $\left\{F_{1}, \ldots, F_{k}\right\}$ such that $F_{1} \succeq_{h r} F_{t}$ for all $t$. The optimal revenue of this setting is at most twice the revenue of Vickrey auction with one extra bidder drawn from $F_{1}$.

Proof. Let $\mathbf{v}$ denote the valuation profile of the initial $n$ bidders and let $v^{*}$ the valuation of the extra bidder from the dominant distribution. Let $R\left(\mathbf{v}, v^{*}\right)$ and $S\left(\mathbf{v}, v^{*}\right)$ denote the winners of the optimal auction $(M)$ and of the second price auction with the extra bidder $\left(S P_{n+1}\right)$ respectively. We will show that the two auctions are commensurate (see [7]) which is sufficient for proving the lemma. Establishing commensurateness boils down to showing that:

$$
\begin{aligned}
& \mathbb{E}_{\mathbf{v}, v^{*}}\left[\phi_{S\left(\mathbf{v}, v^{*}\right)}\left(v_{S\left(\mathbf{v}, v^{*}\right)}\right) \mid S\left(\mathbf{v}, v^{*}\right) \neq R\left(\mathbf{v}, v^{*}\right)\right] \geq 0 \\
& \mathbb{E}_{\mathbf{v}, v^{*}}\left[\phi_{R\left(\mathbf{v}, v^{*}\right)}\left(v_{R\left(\mathbf{v}, v^{*}\right)}\right) \mid S\left(\mathbf{v}, v^{*}\right) \neq R\left(\mathbf{v}, v^{*}\right)\right] \leq \mathbb{E}_{\mathbf{v}, v^{*}}\left[p_{S\left(\mathbf{v}, v^{*}\right)} \mid S\left(\mathbf{v}, v^{*}\right) \neq R\left(\mathbf{v}, v^{*}\right)\right]
\end{aligned}
$$

where $p_{S}$ is the price paid by the winner of the second price auction. The proof of equation (5) is easy and very closely follows the proof in [7] above.

We now prove equation (4). Since $F_{1} \succeq_{h r} F_{t}$ we have that for all $x$ in the intersection of the support of $F_{1}$ and $F_{t}: h_{1}(x) \leq h_{t}(x)$, which in turn implies that $\phi_{1}(x) \leq$ $\phi_{t}(x)$, since $\phi_{t}(x)=x-\frac{1}{h_{t}(x)}$. By the definition of winner in Vickrey auction we have $\forall i: v_{S\left(\mathbf{v}, v^{*}\right)} \geq v_{i}$. In particular, $v_{S\left(\mathbf{v}, v^{*}\right)} \geq v^{*}$. If $v^{*}$ is in the support of $F_{S\left(\mathbf{v}, v^{*}\right)}$, then the latter, by regularity of distributions, implies that $\phi_{S\left(\mathbf{v}, v^{*}\right)}\left(v_{S\left(\mathbf{v}, v^{*}\right)}\right) \geq \phi_{S\left(\mathbf{v}, v^{*}\right)}\left(v^{*}\right)$. Now $F_{1} \succeq_{h r} F_{t}$ implies that $\phi_{S\left(\mathbf{v}, v^{*}\right)}\left(v^{*}\right) \geq \phi_{1}\left(v^{*}\right)$ (since by definition $v^{*}$ must also be in the support of $\left.F_{1}\right)$. If $v^{*}$ is not in the support of $F_{S\left(\mathbf{v}, v^{*}\right)}$, then since $v^{*}<v_{S\left(\mathbf{v}, v^{*}\right)}$ and all the supports are intervals, it must be that $v^{*}$ is below the lower bound $L$ of the support of $F_{S\left(\mathbf{v}, v^{*}\right)}$. Wlog we can assume that the support of $F_{1}$ intersects the support of every other distribution. Hence, since $v^{*}$ is below $L$ and the support of $F_{1}$ is an interval, $L$ will also be in the support of $F_{1}$. Thus $L$ is in the intersection of the two supports. By regularity of $F_{S\left(\mathbf{v}, v^{*}\right)}, F_{1}$ and by the hazard rate dominance assumption, we have $\phi_{S\left(\mathbf{v}, v^{*}\right)}\left(v_{S\left(\mathbf{v}, v^{*}\right)}\right) \geq \phi_{S\left(\mathbf{v}, v^{*}\right)}(L) \geq \phi_{1}(L) \geq \phi_{1}\left(v^{*}\right)$. Thus in any case $\phi_{S\left(\mathbf{v}, v^{*}\right)}\left(v_{S\left(\mathbf{v}, v^{*}\right)}\right) \geq \phi_{1}\left(v^{*}\right)$. Hence, we immediately get that:

$$
\mathbb{E}_{\mathbf{v}, v^{*}}\left[\phi_{S\left(\mathbf{v}, v^{*}\right)}\left(v_{S\left(\mathbf{v}, v^{*}\right)}\right) \mid S\left(\mathbf{v}, v^{*}\right) \neq R\left(\mathbf{v}, v^{*}\right)\right] \geq \mathbb{E}_{\mathbf{v}, v^{*}}\left[\phi_{1}\left(v^{*}\right) \mid S\left(\mathbf{v}, v^{*}\right) \neq R\left(\mathbf{v}, v^{*}\right)\right]
$$

Conditioned on $\mathbf{v}$ the latter expectation becomes:

$$
\mathbb{E}_{v^{*}}\left[\phi_{1}\left(v^{*}\right) \mid S\left(\mathbf{v}, v^{*}\right) \neq R\left(\mathbf{v}, v^{*}\right), \mathbf{v}\right]
$$

But conditioned on $\mathbf{v}, R\left(\mathbf{v}, v^{*}\right)$ is some fixed bidder $i$. Hence, the latter expectation is equivalent to: $\mathbb{E}_{v^{*}}\left[\phi_{1}\left(v^{*}\right) \mid S\left(\mathbf{v}, v^{*}\right) \neq i\right]$ for some $i$. We claim that for all $i$ the latter expectation must be positive. Conditioned on $\mathbf{v}$, the event $S\left(\mathbf{v}, v^{*}\right) \neq i$ happens only if $v^{*}$ is sufficiently high, i.e., there is a threshold $\theta(\mathbf{v})$ such that $S\left(\mathbf{v}, v^{*}\right) \neq i$ happens only if $v^{*} \geq \theta(\mathbf{v})$ (if $i$ was the maximum valued bidder in the profile $\mathbf{v}$ then $\theta(\mathbf{v})=v_{i}$, else $\theta(\mathbf{v})=0$.) By regularity of distributions, $v^{*} \geq \theta(\mathbf{v})$ translates to $\phi_{1}\left(v^{*}\right) \geq \phi_{1}(\theta)$. So we now have to show that: $\mathbb{E}_{v^{*}}\left[\phi_{1}\left(v^{*}\right) \mid \phi_{1}\left(v^{*}\right) \geq \phi_{1}(\theta)\right] \geq 0$. Since the unconditional expectation of virtual value is already non-negative, the expectation conditioned on a lower bound on virtual values is clearly non-negative. 
Examples and Applications. There are many situations where a hazard-rate dominant distribution aactually exists in the market. We provide some examples below.

Uniform, Exponential, Power-law distributions. Suppose the $k$ underlying distributions were all uniform distributions of the form $U\left[a_{i}, b_{i}\right]$. The hazard rate $h_{i}(x)=\frac{1}{b_{i}-x}$. Clearly, the distribution with a larger $b_{i}$ hazard-rate dominates the distribution with a smaller $b_{i}$. If the $k$ underlying distributions were all exponential distributions, i.e., $G_{i}(x)=1-e^{-\lambda_{i} x}$, then the hazard rate $h_{i}(x)=\lambda_{i}$. Thus the distribution with the smallest $\lambda_{i}$ hazard rate dominates the rest. If the $k$ underlying distributions were all power-law distributions, namely, $G_{i}(x)=1-\frac{1}{x^{\alpha_{i}}}$, then the hazard rate $h_{i}(x)=\frac{\alpha_{i}}{x}$. Thus the distribution with the smallest $\alpha_{i}$ hazard-rate dominates the rest.

A general condition. If all the $k$ underlying regular distributions were such that for any pair $i, j$ they satisfy $1-G_{i}(x)=\left(1-G_{j}(x)\right)^{\theta_{i j}}$, then it is easy to verify that there always exists one distribution that hazard-rate dominates the rest of the distributions. For instance, the family of exponential distributions, and the family of power-law distributions are special cases of this general condition.

\section{Non-Targeted Advertising and the i.i.d. Irregular Setting}

In this section we consider the setting where all the bidders are drawn from the same distribution $F$. We assume that $F$ can be written as a convex combination of $k$ regular distributions $F_{1}, \ldots, F_{k}$, i.e. $F=\sum_{t=1}^{k} p_{t} F_{t}$ and such that the mixture probability $p_{t}$ for every distribution is at least some constant $\delta: \forall t \in[1, \ldots, k]: p_{t} \geq \delta$. A natural question to ask in an i.i.d. setting is how many extra bidders should be recruited from the original distribution to achieve a constant fraction of the optimal revenue (i.e., by running a non-targeted advertising campaign)?

In this section answer the above question as a function of the number of underlying distributions $k$ and the minimum mixture probability $\delta$. We remark that our results in this section don't require the decomposition of $F$ into the $F_{t}$ 's resemble the distribution of the underlying population groups. Even if the number of underlying population groups is very large, as long as there is some mathematical way of decomposing $F$ into $k$ regular distributions with a minimum mixture probability of $\delta$, our results go through. Hence, one can optimize our result for each $F$ by finding the decomposition that minimizes our approximation ratio.

Theorem 3. Consider an auction among n i.i.d. irregular bidders where the bidders' distribution $F$ can be decomposed into a mixture of $k$ regular distributions $\left\{G_{1}, \ldots, G_{k}\right\}$ with minimum mixture probability $\delta$. The revenue of the optimal auction in this setting is at most $2 \frac{k+1}{k}$ the revenue of a Vickrey auction with $\Theta\left(\frac{\log (k)}{\delta}\right)$ extra bidders drawn from distribution $F$.

Proof. Suppose that we bring $n^{*}$ extra bidders in the auction. Even if the decomposition of the distribution $F$ doesn't correspond to an actual market decomposition, we can always think of the value of each of the bidders drawn as follows: first we draw a number $t$ from 1 to $k$ according to the mixture probabilities $p_{t}$ and then we draw a value from distribution $G_{t}$. 
Let $\mathcal{E}$ be the event that all numbers 1 to $k$ are represented by the $n^{*}$ random numbers drawn to produce the value of the $n^{*}$ extra bidders. The problem is a generalization of the coupon collector problem where there are $k$ coupons and each coupon arrives with probability $p_{t} \geq \delta$. The relevant question is, what is the probability that all the coupons are collected after $n^{*}$ coupon draws? The probability that a coupon $t$ is not collected after $n^{*}$ draws is: $\left(1-p_{t}\right)^{n^{*}} \leq(1-\delta)^{n^{*}} \leq e^{-n^{*} \delta}$. Hence, by the union bound, the probability that some coupon is not collected after $n^{*}$ draws is at most $k e^{-n^{*} \delta}$. Thus the probability of event $\mathcal{E}$ is at least $1-k e^{-n^{*} \delta}$. Thus if $n^{*}=\frac{\log (k)+\log (k+1)}{\delta}$ then the probability of $\mathcal{E}$ is at least $1-\frac{1}{k+1}$.

Conditional on event $\mathcal{E}$ happening we know that the revenue of the auction is the revenue of the initial auction with at least one player extra drawn from each of the underlying $k$ regular distributions. Thus we can apply our main theorem 1 to get that the expected revenue conditional on $\mathcal{E}$ is at least $\frac{1}{2}$ of the optimal revenue with only the initial $n$ bidders. Thus:

$$
\mathcal{R}_{S P_{n+n^{*}}} \geq\left(1-\frac{1}{k+1}\right) \mathbb{E}_{v, \tilde{v} \sim F^{n+n^{*}}}\left[R_{S P_{n+n^{*}}}(v, \tilde{v}) \mid \mathcal{E}\right] \geq\left(1-\frac{1}{k+1}\right) \frac{1}{2} \mathcal{R}_{M}
$$

Theorem 4. Consider an auction among $n$ i.i.d. irregular bidders where the bidders' distribution $F$ can be decomposed into a mixture of $k$ regular distributions $\left\{G_{1}, \ldots, G_{k}\right\}$ such that $G_{1}$ hazard rate dominates $G_{t}$ for all $t>1$. The revenue of the optimal auction in this setting is at most $2 \frac{e}{e-1}$ the revenue of a Vickrey auction with $\frac{1}{p_{1}}$ extra bidders drawn from distribution $F$.

Proof. Similar to theorem 3 conditional on the even that an extra player is drawn from the hazard rate distribution, we can apply Lemma1 to get that this conditional expected revenue is at least half the optimal revenue with the initial set of players. If we bring $n^{*}$ extra players then the probability of the above event happening is $1-\left(1-p_{1}\right)^{n^{*}} \geq$ $1-e^{-n^{*} p_{1}}$. Setting $n^{*}=\frac{1}{p_{1}}$ we get the theorem.

Prior-Independent Mechanisms. The two theorems above imply prior-independent mechanisms for the i.i.d. irregular setting based on a reasoning similar to the one used by [5] in converting Bulow-Klemperer results to prior-independent mechanims in the i.i.d. regular setting. Specifically, instead of bringing $k$ extra i.i.d. bidders we could use the maximum value of a random subset of $k$ existing bidders as a reserve on the remaining $n-k$ bidders. The theorems above then imply that this prior-independent mechanism yields a constant approximation with respect to the optimal mechanism among the $n-k$ bidders. Further, since the bidders are all i.i.d., and the $k$ bidders were chosen before their valuation are drawn, the expected optimal revenue among the $n-k$ bidders is at least $1-\frac{k}{n}$ of the optimal revenue among the $n$ bidders. Thus as long as the number of bidders $k$ required by Theorems 3 and 4 is smaller than $n$, this approach yields a priorindependent mechanism with a meaningful revenue approximation guarantee. Hence, Theorem 3 implies that if $n \delta \geq c \log (k)$ (i.e. the expected number of players from each population is at least $c \log (k))$ the random sampling mechanism described above is $2 \frac{k+1}{k} \frac{c}{c-1}$ approximate. Similarly, Theorem 4 implies that it is $2 \frac{e}{e-1} \frac{c}{c-1}$-approximate, if $n \cdot p_{1} \geq c$, i.e. the expected number of players from the hazard-rate dominant distribution at least $c$. 


\section{Vickrey with Single Reserve for Irregular Settings}

In this section we prove revenue guarantees for Vickrey auction with a single reserve in the general irregular setting.

Theorem 5. Consider an auction among $n$ non-i.i.d irregular bidders where each bidder's distribution $F_{i}$ is some mixture of $k$ regular distribution $\left\{G_{1}, \ldots, G_{k}\right\}$ (the set of regular distributions is the same for all bidders but the mixture probabilities could be different). The revenue of the optimal auction in the above setting is at most $4 k$ times the revenue of a second price auction with a single reserve price which corresponds to the monopoly reserve price of one of the $k$ distributions $G_{i}$.

Proof. We use the same notation as in Section 3 . In particular, we let $q$ denote the index profile of distributions for all players and $p(q)=\prod_{i=1}^{n} p_{i, q_{i}}$ be the probability that an index profile arises. Let $G(q)=\times_{i} G_{q_{i}}$ be the product distribution that corresponds to how players values are distributed conditional on the coin tosses having value $q$.

Let $M_{q}$ be the optimal mechanism when bidders' distribution profile is $q$. Let $\mathcal{R}_{M}^{q}$ be the expected revenue of mechanism $M_{q}$. By equation (2) in Section 3 we have,

$$
\mathcal{R}_{M} \leq \sum_{q \in[1 . . k]^{n}} p(q) \mathbb{E}_{\mathbf{v} \sim G(q)}\left[R_{M}^{q}(\mathbf{v})\right] \leq 2 \sum_{q \in[1 . . k]^{n}} p(q) \mathbb{E}_{\mathbf{v} \sim G(q)}\left[R_{S P_{n+k(q)}}(\mathbf{v})\right]
$$

Consider the auction $S P_{n+k(q)}$. If instead of adding the $k(q)$ extra bidders, we place a random reserve drawn from the distribution of the maximum value among the $k(q)$ extra bidders, and ran the Vickrey auction. Call the later $S P_{n}(R(q))$. If the winner of the auction $S P_{n+k(q)}$ is one among the original $n$ bidders, clearly $S P_{n+k(q)}$ and $S P_{n}(R(q))$ will have the same expected revenue. Further, the expected revenue of $S P_{n+k(q)}$ conditioned on the winner being one among the original $n$ bidders is no smaller than the expected revenue of $S P_{n+k(q)}$ conditioned on the winner being one among the newly added $k(q)$ bidders. Also, the probability that the winner comes from the newly added $k(q)$ bidders is at most $1 / 2$. Thus $S P_{n}(R(q)) \geq \frac{1}{2} S P_{n+k(q)}$. Combining this with Equation (6), we have

$$
\begin{aligned}
\mathcal{R}_{M} & \leq 2 \sum_{q \in[1 . . k]^{n}} p(q) \mathbb{E}_{\mathbf{v} \sim G(q)}\left[R_{S P_{n+k(q)}}(\mathbf{v})\right] \leq 4 \sum_{q \in[1 \ldots k]^{n}} p(q) \mathbb{E}_{\mathbf{v} \sim G(q)}\left[R_{S P_{n}(R(q))}(\mathbf{v})\right] \\
& =\sum_{t=1}^{k} 4 \sum_{q \in[1 . . k]^{n}} p(q) \mathbb{E}_{\mathbf{v} \sim G(q)}\left[R_{S P_{n}(R(q), t)}(\mathbf{v})\right] \\
& \leq 4 k \sum_{q \in[1 . . k]^{n}} p(q) \mathbb{E}_{\mathbf{v} \sim G(q)}\left[R_{S P_{n}\left(R(q), t^{*}\right)}(\mathbf{v})\right]
\end{aligned}
$$

In equation (7), the revenue $R_{S P_{n}(R(q))}(\mathbf{v})$ is written as $\sum_{t=1}^{k} R_{S P_{n}(R(q), t)}(\mathbf{v})$, i.e., as the sum of contributions from each population group. Given this split, there exists a polulation group $t^{*}$ that gets at least $\frac{1}{k}$ fraction of all groups together, and thus at least $\frac{1}{4 k}$ fraction of the optimal mechanism, which is what is expressed through inequality [8]. 
Now the auction $S P_{n}(R(q))$ from the perspective of the group $t^{*}$ is just the Vickrey auction run for group $t^{*}$ alone with a single random reserve of $\max \{R(q)$, Maximum value from groups other than $\left.t^{*}\right\}$. However within the group $t^{*}$ since we are in a i.i.d regular setting it is optimal to run Vickrey auction for the group $t^{*}$ alone with the monopoly reserve price of that group. That is if we replace the single reserve of $\max \left\{R(q)\right.$, Maximum value from groups other than $\left.t^{*}\right\}$ with the optimal (monopoly) reserve price for $t^{*}$, Vickrey auction for group $t^{*}$ with such a reserve gives no lesser revenue, and this holds for every $q$ ! Finally, when we add in the agents from other groups, single-item Vickrey auction's revenue for the entire population with monopoly reserve price of group $t^{*}$ is no smaller than the revenue of single-item Vickrey auction for group $t^{*}$ alone with the monopoly reserve price of group $t^{*}$. Chaining the last two statements proves the theorem.

\section{References}

[1] Bulow, J. And Klemperer, P. 1996. Auctions vs negotiations. American Economic Review 86, 1, 180-194.

[2] Chawla, S., Hartline, J. D., And Kleinberg, R. D. 2007. Algorithmic pricing via virtual valuations. In ACM Conference on Electronic Commerce. 243251.

[3] Chawla, S., Hartline, J. D., Malec, D. L., And Sivan, B. 2010. Multiparameter mechanism design and sequential posted pricing. In STOC. 311-320.

[4] Devanur, N. R., Hartline, J. D., Karlin, A. R., And Nguyen, C. T. 2011. Prior-independent multi-parameter mechanism design. In WINE. 122-133.

[5] Dhangwatnotai, P., Roughgarden, T., And Yan, Q. 2010. Revenue maximization with a single sample. In Proceedings of the 11th ACM conference on Electronic commerce. EC'10. 129-138.

[6] Guimaraes, B. And Sheedy, K. D. 2011. Sales and monetary policy. American Economic Review 101, 2, 844-76.

[7] Hartline, J. D. And Roughgarden, T. 2009. Simple versus optimal mechanisms. In ACM Conference on Electronic Commerce. 225-234.

[8] Johnson, J. P. AND MYATT, D. P. 2003. Multiproduct quality competition: Fighting brands and product line pruning. The American Economic Review 93, 3, pp. $748-774$.

[9] KirKegaARD, R. 2011. Ranking asymmetric auctions using the dispersive order. Manuscript.

[10] Myerson, R. 1981. Optimal auction design. Mathematics of Operations Research 6, 58-73.

[11] NeEman, Z. 2003. The effectiveness of english auctions. Games and Economic Behavior 43, 2, 214-238.

[12] Robinson, J. 1933. The Economics of Imperfect Competition. McMillan and Co.

[13] Ronen, A. 2001. On approximating optimal auctions. In ACM Conference on Electronic Commerce. 11-17.

[14] Roughgarden, T., TAlgam-Cohen, I., And Yan, Q. 2012. Supply-limiting mechanisms. In ACM Conference on Electronic Commerce. 844-861. 